\title{
High-pressure behavior of dense hydrogen up to 3.5 TPa from density functional theory calculations
}

\author{
Hua Y. Geng, Hong X. Song, J. F. Li, and Q. Wu \\ National Key Laboratory of Shock Wave and Detonation Physics, Institute of Fluid Physics, \\ CAEP; P.O.Box 919-102 Mianyang, Sichuan, P. R. China, 621900
}

\begin{abstract}
Structural behavior and equation of state of atomic and molecular crystal phases of dense hydrogen at pressures up to $3.5 \mathrm{TPa}$ are systematically investigated with density functional theory. The results indicate that the Vinet EOS model that fitted to low-pressure experimental data overestimates the compressibility of dense hydrogen drastically when beyond $500 \mathrm{GPa}$. Metastable multi-atomic molecular phases with weak covalent bonds are observed. When compressed beyond about 2.8 TPa, these exotic low-coordinated phases become competitive with the groundstate and other high-symmetry atomic phases. Using nudged elastic band method, the transition path and the associated energy barrier between these high-pressure phases are evaluated. In particular for the case of dissociation of diatomic molecular phase into the atomic metallic Cs-IV phase, the existent barrier might raise the transition pressure about $200 \mathrm{GPa}$ at low temperatures. Plenty of flat and broad basins on the energy surface of dense hydrogen have been discovered, which should take a major responsibility for the highly anharmonic zero point vibrations of the lattice, as well as the quantum structure fluctuations in some extreme cases. At zero pressure, our analysis demonstrates that all of these atomic phases of dense hydrogen known so far are unquenchable.
\end{abstract}

PACS numbers: 67.80.F-, 62.50.-p, 64.10.+h, 71.15.Nc, 64.70.K-, 71.30.+h

Keywords: dense hydrogen, phase transition, equation of state, high pressure, phase stability 


\section{INTRODUCTION}

Having only a single electron outside the nucleus, hydrogen is the simplest and most abundant element in the universe. It is also an essential element for models of stellar and planetary interiors. $\underline{1}_{2}^{2}$ Hydrogen shows characteristics of both the group I alkalis and the the group VII halogens. At low pressures, hydrogen isotopes are halogenous, covalent diatomic molecules that form insulators. Yet at high pressures, it is one of the most difficult to understand. It displays anomalous melting behavior with a maximum in the melting temperature versus pressure curve at high temperatures, $\frac{3,4}{\underline{4}}$ and undergoes a first-order liquid-liquid transition under further compression. $.^{5}, \underline{6}$ At low temperatures, it is experimentally known that hydrogen can exist as a rotational crystal (phase I) on a hexagonal close-packed (HCP) lattice to high pressures $(P<110 \mathrm{GPa})$, followed by a transition into the broken-symmetry phase II $(110 \mathrm{GPa}<P<150 \mathrm{GPa})$ which is marked by a change to wide-angle libration and hence to a continuing incoherence of motion between different molecules, and then to phase III at about $150 \mathrm{GPa} .^{\underline{7}}$ Possible pressure-induced insulator-metal transition also has been extensively studied up to $320 \mathrm{GPa} \cdot \underline{\underline{7-10}}$ However, beyond the fact that protons remain paired within this pressure range, their time-average locations are to date experimentally unknown, mainly because hydrogen atoms scatter X-rays only weakly, leading to low-resolution diffraction patterns. $\underline{7} \underline{\underline{11}}$ Experimental data at higher pressures are scarce, and insightful understanding of structural behavior of ultra-dense hydrogen is lacking.

Theoretical prediction of stable crystalline structures and properties of dense hydrogen at high pressures has been pursued for decades. ${ }^{12} \underline{-19}$ It is difficult because of the need to search the very large space of possible structures, and the necessity of obtaining accurate energies for each of these structures. 17,19 First-principles density functional theory (DFT) has been proved as an efficient approach of calculating quite accurate energies, and has provided insights into properties of various materials, including solid hydrogen under compressions. At present, DFT offers a high level of theoretical description at which we can carry out searches over many possible candidate structures $\underline{\underline{16}} \underline{19}$ Recent DFT calculations have predicted that within the static-lattice approximation, the most stable phases of dense hydrogen are $P 6_{3} / m$ (<105 GPa), C2/c (105-270 GPa), Cmca-12 (270-385 GPa), and Cmca (385-490 GPa), followed by atomic $I 4_{1} /$ amd (Cs-IV) phase, $\stackrel{17,18}{\underline{10}}$ and then $R 3 m$ (or $\left.R \overline{3} m\right) ! \underline{19}$

On the other hand, at pressures high enough so that electrons are fully ionized out to form 
a uniform background, hydrogen becomes one-component plasma. ${ }^{20}$ In this regime the dominant Coulomb interaction forces the ions into an ordered configuration called Wigner crystal, and stabilizes in a body-centered cubic (BCC) structure $\stackrel{20-22}{\underline{20}}$ Except this, the general structural and compressional behavior of dense hydrogen at ultra-high pressures beyond several Mbar (1 Mbar=100 GPa) are still poorly understood, in spite of a few theoretical investigations available. $\underline{17}, \underline{19}$ In particular, the energy barriers of pressure-induced phase transitions are completely unknown, especially that of molecule dissociation. A high energy barrier would lead to a hysteresis and raise the transition pressure from what it otherwise would be. $\frac{23}{}$ Another important issue that has not been solved completely is about the applicability of extrapolating the equation of state (EOS) that fitted at low pressure to higher pressures. This is an intriguing problem because we knew that the EOS model fitted to lower-pressure data (up to $42 \mathrm{GPa})^{7}$ fails to capture (underestimates) the compressibility of $\mathrm{H}_{2}$ and $\mathrm{D}_{2}$ at the relatively higher pressures, $\underline{\underline{24}}$ which is due to the transition of $\mathrm{H}_{2}\left(\right.$ or $\left.\mathrm{D}_{2}\right)$ from a freely rotating phase into a broken-symmetry phase. At ultra-high pressures, diatomic hydrogen dissociates into atomic phase, and this transition might invalidate the early established EOS model again very likely.

The groundstate structures of atomic metallic hydrogen from $500 \mathrm{GPa}$ to $4.5 \mathrm{TPa}$ has been extensively searched by McMahon et al $\underline{\underline{19}}$ using the ab initio random structure searching (AIRSS) method $\underline{25}^{2}$ Their work was carried out for unit cells containing only 4 and 6 atoms. However, as recent investigations suggested, complex structures can be adopted by simple alkali metals at high pressures $\stackrel{26}{227}$ Similar phenomenon might also occur in dense hydrogen. Furthermore, there is no absolute guarantee for a specific structure searching approach to find the true ground state within a finite computational time due to the limited phase space it can explore. There are examples that AIRSS calculations failed to detect lower energy states which later were captured by other structure searching method such as evolutionary algorithms. $\stackrel{27,28}{2}$ Therefore a cross-check of the proposed groundstate structures with alternative methods is always necessary to ascertain the results. With systematic and accurate first-principles calculations using evolutionary searching method combined with particle swarm optimization algorithm, $\frac{29}{\underline{a}}$ we confirm in this work that Cs-IV atomic phase with a space group of $I 4_{1} /$ amd becomes the most stable phase beyond $490 \mathrm{GPa}$, but a novel structure $F d d d$ is also found to be degenerate with it over a wide range of pressure. These two metallic phases persist up to $2.3 \mathrm{TPa}$, where exotic multi-atomic structures with low 
symmetry becomes competitive. Our calculation also indicates that dense hydrogen has many very broad and flat basins on the energy surface, which has never been noticed before. This flatness of the energy variation not only leads to great anharmonicity in zero-point vibrations of lattice, but also blurs the boundary of some crystal structures. Furthermore, the transition paths between high pressure phases are modeled with nudged elastic band (NEB) method, and the associated enthalpy barriers and energy variations are analyzed. In the next section we will present the methods for total energy and NEB calculations. The results and discussion are given in Sec. III, followed by a summary in Sec. IV.

\section{METHODOLOGY}

\section{A. Total energy calculation}

The total energy calculations were performed with DFT as implemented in the Vienna Ab-initio Simulation Package (VASP) $\stackrel{30}{n}$ The electronic structure was described with all-electron like projector augmented-wave (PAW) potential, $\stackrel{31,32}{ }$ and the Perdew-BurkeErnzerhof (PBE) exchange-correlation functional was used. ${ }^{33}$ A hard version of the PAW potential that is specially designed for high pressure applications was employed. The k-point sets were generated separately for each unit cell encountered during the procedure, and a high quality Brillouin zone sampling with a grid of $15 \times 15 \times 15$ were found to be sufficient for structure optimization. When re-calculate the enthalpy curves, we used a denser k-point mesh that can generate at least 1500 irreducible points. The residual Pulay stress was removed by increasing the kinetic energy cutoff of the plane wave basis set to $900 \mathrm{eV}$, which was confirmed by observing the vanishment of the difference between the HellmannFeynman pressure and that computed from the energy curve with $P=-d E / d V$. Increase the energy cutoff to $1200 \mathrm{eV}$ led to a pressure change less than $0.1 \mathrm{GPa}$. A justification of the methods, especially DFT and the employed pseudopotential, is given in appendix. With this parameter setting, the structural features are estimated to be converged to better than $0.2 \%$, the convergence of total energy is to within $3 \mathrm{meV}$ per proton, and the relative energy difference between structures is to within $1 \mathrm{meV}$ per proton.

By minimizing the enthalpy, we carried out extensive and systematic structure searches of molecular and atomic phases at pressures up to 3.5 TPa. All structures were fully relaxed 
at fixed volumes with a force tolerance of $0.1 m \mathrm{eV}^{-1}$. The pressure was directly computed with the Hellmann-Feynman theorem, which then was used to calculate the enthalpy. In addition to diatomic molecular phases, many high-pressure candidates of atomic phase were considered and their enthalpies were calculated, including cubic structures (SC, BCC, and FCC) and their low-symmetry distortions ( $\beta$-Po, $\beta$-Hg, In-I, and In-II), hexagonal structures (HCP and $\omega$-Ti), diamond structure, $I 4_{1} /$ amd ( $\beta$-Sn and Cs-IV) phases, and so on. $\underline{34}^{\text {Details }}$ are presented in the following subsections.

\section{B. Structure search}

The search for high pressure structures of dense hydrogen was mainly carried out with the particle swarm optimization (PSO) technique ${ }^{29}$ within an evolutionary scheme that combined with first-principles total energy calculations using VASP. PSO is an efficient approach of evolutionary methodology but quite different from genetic algorithm (GA). In particular the major evolution operations of crossover and mutation in GA have been avoided. PSO has been verified to perform well on many optimization problems. $\underline{26}, 27,29$ In this work, stable and meta-stable structures containing up to 24 atoms per unit cell at pressures up to $3.5 \mathrm{TPa}$ were automatically explored and generated by CALYPSO code $\stackrel{29}{,}$ which implements PSO algorithm. With a population size of 30 in each generation and a total allowed number of generations of 30 , it is sufficient to ensure the convergence of the structure search.

As a complement to the CALYPSO search, in order to better understand the relationship between symmetry and the structure stability, as well as to track the evolution of structures with pressure, we also performed local structure searches manually. It was based on the already known information about stable and meta-stable phases at relatively low pressures. $\stackrel{16}{17}$ Some molecular and atomic phases which were not yet fully investigated previously were added into the structure library of search. The atomic phases were selected from

Ref 34 by consulting previous theoretical predictions to pick out the most likely candidates, while putting high weight on low-symmetric structures.

The initial structures for optimization were generated from the search library by drifting the ionic positions and distorting the lattice shape randomly. Interpolated configurations between the most stable phases were also taken into account. Although most of the phases 
in the search library are locally stable, with large enough distortions new structures can always be produced. To eliminate a possible sensitive dependence of the final results on the initial configurations, the ionic relaxation procedure employed algorithms of both quasiNewton and conjugate-gradient method alternatively. The derived structures were then fully relaxed to the energy minima at constant volumes without any symmetry restrictions. After the ionic optimization process converged, we calculated the pressure and then the corresponding enthalpy. At each pressure, the search process was repeated several times (each iteration involves all structures in the search library that was updated accordingly) to confirm the results, and in total 4 typical pressure values (around 0.5, 1, 1.5 and $2 \mathrm{TPa}$, respectively) were explored. The whole enthalpy curves of the low-lying phases were then computed.

\section{NEB calculation}

Structural phase transition paths and the associated energy barriers were modeled using the nudged elastic band (NEB) method ${ }^{35}$ as implemented in the VASP code, which searches for the minimum energy path (MEP) by moving a chain of ionic configurations or images that bridges the initial and the target structures. Tangential springs were introduced to keep the images being equidistant during the relaxation. The potential energy maximum along the MEP is the saddle point energy which gives the activation energy barrier.

In calculations, the supercell method was employed, which in most cases contains 12 atoms, and in some special situations a cell with 24 atoms was also used. To model the transition path with a variable cell volume and shape using NEB technique, the continuous MEP was constructed by linking the images with springs only in a pre-aligned supercell (i.e., the images due to periodic boundary conditions were discarded). When computing the transition path from high-pressure dense phases to a diatomic molecular phase at $0 \mathrm{GPa}$, the molecular structure was generated by distorting and then relaxing the corresponding dense hydrogen phase respectively. This strategy simplifies the NEB calculations greatly since in this way the initial path generated through a linear interpolation of the initial and the target configurations gives a good approximation to the final transition path. Then a minimization of the whole system was carried out to trace out the MEP. In all calculations at least five images (seven if includes the two end images) were employed, which in most 
cases is sufficient to give an acceptable resolution to the discrete representation of the MEP.

\section{Calculation of phonons in harmonic approximation}

Zero-point (ZP) vibrations of protons were neglected during the structure search and optimization procedure, but with a subsequent estimation of its impact on the relative stabilities using harmonic ZP energy (ZPE) calculations. The full phonon spectra in a harmonic approximation were calculated with the small-displacement method as implemented in the PHON code. ${ }^{36}$ Big enough supercells containing more than 64 atoms were used. In the associated DFT calculations, a Brillouin zone sampling mesh of $20 \times 20 \times 20$, a kinetic energy cutoff of $1000 \mathrm{eV}$, and a very dense support augmentation charge grid that is required for an accurate force calculation were used. This setup gives a convergence in the ZPE better than $2 \mathrm{meV}$ per proton. The magnitude of the small displacement was slightly varied to check the numerical stability of the calculated force constant matrices. The ZPE was estimated from the phonon density of states $g(\omega)$ by $\int g(\omega) \hbar \omega / 2 d \omega$, and the ZP mean square displacement (MSD) was calculated by $\int g(\omega) \hbar /(2 M \omega) d \omega$, where $M$ is the mass of hydrogen atom.

\section{RESULTS AND DISCUSSION}

\section{A. Static structure and enthalpy}

\section{Ground-state of dense hydrogen}

The calculated enthalpy difference with respect to a reference state (virtually defined by its enthalpy as $H=-3.985+0.216 P^{0.55} \mathrm{eV} /$ proton, $P$ in a unit of GPa $)^{37}$ of the most stable phases are shown in Fig 1, Overall, our results agree very well with previous theoretical predications: diatomic $C 2 / c$ is the groundstate at $110 \mathrm{GPa}$ and transforms into $\mathrm{Cmca}-12$ at about $255 \mathrm{GPa} ; \stackrel{17}{\underline{18}}$ at $370 \mathrm{GPa}, \mathrm{Cmca}$ overtakes slightly, $\stackrel{17}{ }$ but it soon degenerates into Cmca-12, which is stable until an atomic phase- Cs-IV with space group $I 4_{1} / a m d^{16}$ and its distortion $F d d d$ - becomes the groundstate at $495 \mathrm{GPa}$; beyond $2.3 \mathrm{TPa}$, the most favored phase is shifted to trigonal $R 3 m$ in a static lattice approximation. $\underline{19}$

The main transition points are indicated by arrows in Fig.1. Point B is an intersection of the molecular phases and the low-lying atomic Cs-IV and Fddd phases. Namely, 


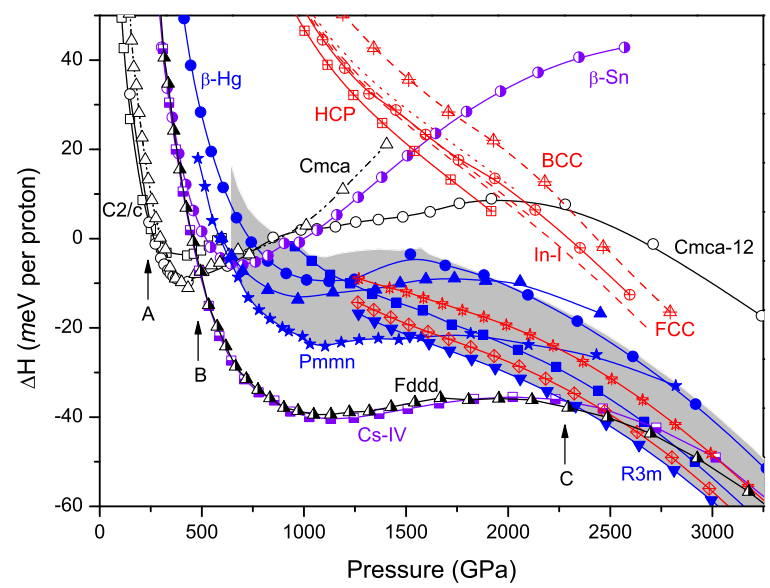

FIG. 1. (color online) Enthalpy difference per proton as a function of pressure with respect to a reference state. Arrows indicate the main phase transitions of the ground state and the shadow marks the region where the meta-stable low-symmetry multi-atomic phases lie in. Typical curves are labeled as follows: open square $C 2 / c$, open circle- $C m c a-12$, open triangle $C m c a$, filled star-Pmmn, filled square- $C 2 / m(2)$, filled up-triangle- $C 2 / m(1)$, filled circle- $\beta$-Hg, half-filled square-Cs-IV, half-filled triangle- $F d d d$, filled down-triangle- $R 3 m$, crossed rhombus $-R \overline{3} m$, crossed pentagram- $-P 6_{3} / m m c$, crossed triangle-BCC, crossed circle-FCC, and crossed square-HCP.

it is a pressure-induced dissociation point. This is in agreement with previous DFT calculations, $\underline{16,17,19}$ except for a newly discovered degenerate phase $F d d d$ that was not detected before. $F d d d$ is an orthorhombic variant of the diamond phase, but is also close to being a distortion of Cs-IV locally. Beyond the transition point $\mathrm{C}$, there are many structures with closely competitive enthalpy, reflecting the frustration among competing factors. In this regime, a small change in interactions will tip the balance from favoring one phase into another. Although $R 3 m$ seems to have the lowest static lattice enthalpy, harmonic phonon calculations indicated that the zero point vibrations of lattice might make $R \overline{3} \mathrm{~m}$ be more favored. ${ }^{19}$ It is interesting to note that both Cs-IV and Fddd have 4 nearest neighbor (NN) atoms, and $R \overline{3} m$ has 6 as its first coordination number $(\mathrm{CN})$. In contrast, $R 3 m$ apparently has just $2 \mathrm{NNs}$, but since the distance from its first NN shell to the next one is very short $(0.06 \AA$ at $3 \mathrm{TPa})$, so that it in fact has 6 atoms in its first coordination shell. From this point of view, the evolution of the groundstate structures of dense hydrogen under compression is evident: with increasing density, the structure evolves from with $1 \mathrm{NN}$ (diatomic molecular phases) to that with $4 \mathrm{NNs}$ (atomic Cs-IV and $F d d d)$, and then to that with $6 \mathrm{NNs}(R 3 m$ 


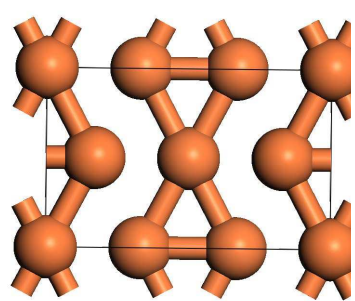

(a)

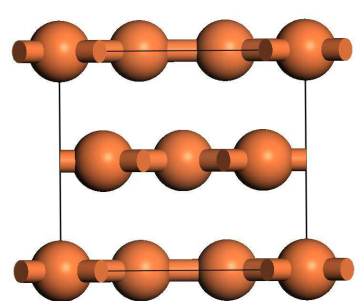

(b)

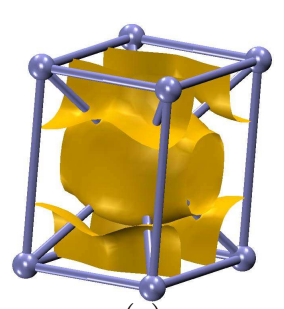

(c)

FIG. 2. (color online) Structure of (a) $C 2 / m(2)$, (b) $C 2 / m(1)$, and (c) $\beta$-Hg. A charge density isosurface also illustrates in (c). The two $C 2 / \mathrm{m}$ phases (both in $I m m m$ representation and projected onto the same plane) are different only in the connection manner of the covalent bonds, and $\beta-\mathrm{Hg}$ is derived from BCC structure with covalent bonds formed along the shortest $c$ direction.

or $R \overline{3} m$ ), finally transforms to cubic structures with 8 (BCC) or 12 (FCC) NNs. ${ }^{19}$

\section{Metastable multi-atomic molecular crystals}

Other monatomic phases with high symmetry, such as BCC, FCC, and HCP, and their distortions, do not approach the ground-state line before 3.5 TPa (the simple cubic (SC) phase is always $50 \mathrm{meV}$ higher than others and not shown here). One interesting phase is $\beta-\mathrm{Hg}$, which caught no attention before. Its enthalpy is already low enough at $600 \mathrm{GPa}$ as shown in Fig,1, and approaches gradually to the groundstate line all the way beyond $3 \mathrm{TPa}$. It might coexist with other meta-stable phases such as Pmmn and $C 2 / m$ over a wide range of pressure. In conventional sense it should be a monatomic phase distorted from BCC structure with a shorter lattice length in the $c$ direction. ${ }^{34}$ But for hydrogen at pressures beyond $600 \mathrm{GPa}$ this lattice length is already short enough so that covalent bonds are established along this direction and it becomes a chained molecular state (see Fig 2(c)]. In fact it is exactly the formation of this kind of multi-atomic molecular bonds that stabilizes the structure, due to a combined effect of pressure induced excitation of the $1 s$ electrons (which weakens the diatomic covalent bond) and the tendency to overlap electronic orbitals among neighboring atoms. It can be seen much clearly by comparing the enthalpy with that of $C m c a$ (diatomic bonds), Pmmn (triatomic bonds, see below), and $C 2 / m$ (molecular chains). Specifically, $\beta$-Hg constitutes linear chains of $\mathrm{H}_{2}$ bonds, whereas $C 2 / m$ consists of chains of $\mathrm{H}_{3}$ clusters. $C 2 / m(1)$ and $C 2 / m(2)$ belong to the same space group. The only difference is in the linkage pattern of the bonds and the chain orientation. In addition, 


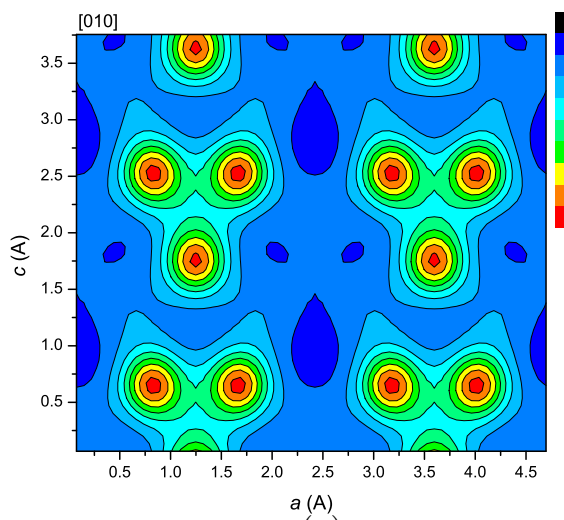

(a)

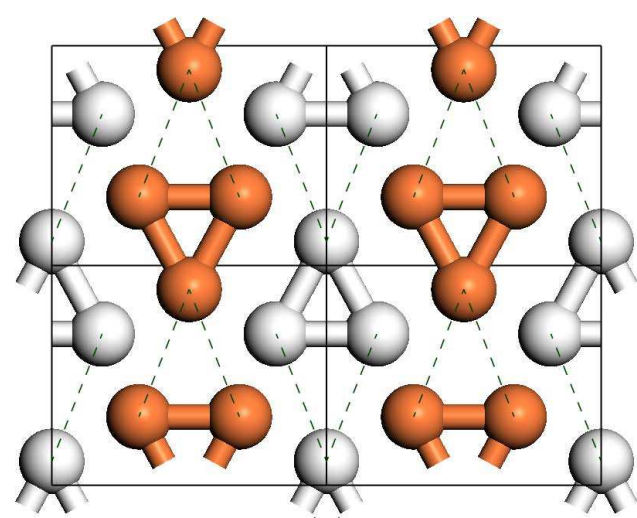

(b)

FIG. 3. (color online) Charge density on [010] plane (a) and the atomic structure (b) of a $2 \times 1 \times 2$ supercell of Pmmn phase at $1104 \mathrm{GPa}$, in which the $\mathrm{H}_{3}$ clusters are evident. In (b): atoms on alternating layers are distinguished by different color/grey scale, and the dashed lines indicate the connection manner of covalent bonds in a chained molecular phase $C 2 / m(2)$.

$C 2 / m(1)$ has a shorter interchain distance and trends to form a bond network, whereas $C 2 / m(2)$ has more localized bonds and thus its structure is much distinct and well defined. Figures 2 33 and table 1 in appendix provide detailed structural information about these phases. It is worthwhile to point out that the $C 2 / m$ phase reported in Ref 19 corresponds to the $C 2 / m(1)$ here, and $C 2 / m(2)$ has a lower enthalpy at high pressures. The existence of this isomorph (and others alike) indicates the complex nature of the structure of dense hydrogen.

Although Pmmn is a triatomic phase (see Fig, 3) and $C 2 / m(2)$ is a chained molecular structure (see Fig 2(a) , they are closely related. Both phases can be drawn on an orthorhombic lattice, and their structural relationship manifests clearly in an $1 \mathrm{mmm}$ representation. By comparing Figs. 3(b) and 2(a) (and also their charge densities), it is easy to find the similarity of these two structures: by compressing the former phase along its $c$ direction to create new bonds along the dashed lines as indicated in figure 3(b) meanwhile shifting the relative position of the alternating layers slightly along this direction, one gets the latter phase. This is actually how the structural transition from Pmmn to $C 2 / m(2)$ takes place at $1.9 \mathrm{TPa}$. It is reasonable since compression reduces inter-distance among $\mathrm{H}_{3}$ clusters, and thus could establish chains of covalent bonds along certain direction if the strain tensor is anisotropic.

It is worthwhile to note that at the intermediate pressure range the NN number of $C 2 / m$ 
phases is about 3, and both Pmmn and $\beta$-Hg have a NN number of 2 . They all are multiatomic molecular phases. Furthermore, distorting some simple high-symmetric structures can make them continuously relax to configurations with a low $\mathrm{CN}$, and the resultant enthalpy lies in the shaded range as shown in Fig.1. The above mentioned multi-atomic molecular phases are members of these low-symmetric structures. This connection provides a plausible answer to the questions of why structures with a CN of 2 or 3 absent from the groundstates, and why dense hydrogen dissociates into orthorhombic atomic Cs-IV instead of simple high-symmetric monatomic phases such as BCC, FCC, or HCP. As it is well known, hydrogen has just one electron outside the nucleus. This makes the electron cloud be compact and tightly attached to the nuclei even at high pressures. On the other hand, at a given density, high-symmetric monatomic phases always have a greater interatomic distance, making it difficult for hydrogen to share electron with its neighbors. In this sense, distortion

of the structure to reduce interatomic distance between some atoms can effectively facilitate wavefunction overlapping and lower the energy. That is the reason why dense hydrogen does not dissociate into simple monatomic phases directly. From another point of view, diatomic covalent bond has been weakened greatly by compression at high pressures, and thus prevents a further transition of these low-symmetric phases into a diatomic molecular configuration via mechanism- for example, the Peierls instability $\underline{38}$ - from happening when the pressure is higher than $500 \mathrm{GPa}$. However, because each hydrogen has only one electron, it is not easy to form stable multi-atomic (triatomic or molecular chain) bonds. Even with the aid of a small portion of $p$ electrons that are excited from $s$ orbitals by compression (this increases the anisotropy of the electron cloud and thus favors multi-atomic bonds), the multi-atomic molecular phases are just be meta-stable, and the balance can be easily tipped down towards the atomic phases with a low $\mathrm{CN}$, which takes advantages of all competing factors. That explains why no groundstate of dense hydrogen has a NN number of 2 or 3.

\section{Electronic structure}

Figure 3(a) shows the calculated charge density of Pmmn at about $1 \mathrm{TPa}$. The triatomic molecules are evident. Note that the environmental charge density is nonzero and the multi-atomic molecules are in fact submerging in a sea of electrons that mediates metallic interactions. Figure 4 plots the electron localization function ${ }^{39.40}$ of $C 2 / m(2)$ at $2.7 \mathrm{TPa}$, 


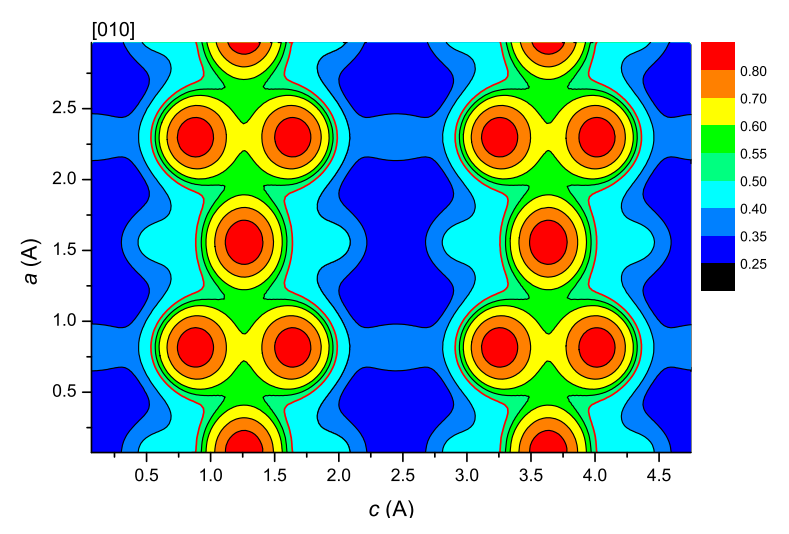

FIG. 4. (color online) Electron localization function of the $C 2 / m(2)$ phase (in a tetragonal $I m m m$ representation) at about 2.7 TPa. Formation of the molecular chains is evident.

where the weak covalent bonds along the molecular chains built up of $\mathrm{H}_{3}$ clusters are distinct. It is important to point out that although $C 2 / m$ and $\beta$ - $\mathrm{Hg}$ are chained molecular phases, they are natural conductors. We do not need to perform a band structure calculation to confirm this, since these phases have an odd number of electrons in their primitive cell, therefore there always have a half-filled band. Although Pmmn has 6 electrons in its primitive cell, a DFT calculation with GGA showed that it is metallic when pressure is beyond $600 \mathrm{GPa}$. This is different from lithium or sodium where intermediate compression induces localization of electrons at interstitial regions and leads to metal-semiconductor-metal transition. $, 27,41,42$

The electronic density of states (DOS) of $C 2 / c, C m c a-12$, Cs-IV, and $C 2 / m(2)$ phases at selected pressures are shown in Fig 5, respectively. Being consistent with previous studies, the molecular hydrogen at $299 \mathrm{GPa}$ does not show metallic characteristics, and there is an energy gap presented at the Fermi level. 17 At a pressure of $490 \mathrm{GPa}$ in a diatomic molecular Cmca-12 phase, the gap already closes up and the material becomes metallic. Here we didnot attempt to determine the precise insulator-metal transition pressure in the molecular phase but instead focused on the general trend of the variation of DOS with pressure. It is interesting to note that the DOS dips down at the Fermi level in Cmca-12, clearly showing that the closure of the gap is due to band overlapping. This feature disappears after transition into the atomic Cs-IV at the same pressure, it also absents from $C 2 / m(2)$ at about 2.7 TPa in spite of covalent bonds presenting in this meta-stable phase. Overall, with increase of the pressure, the localized covalent states in diatomic molecular phases become dispersive and extend towards both the high and low energy ends, which then closes up the 


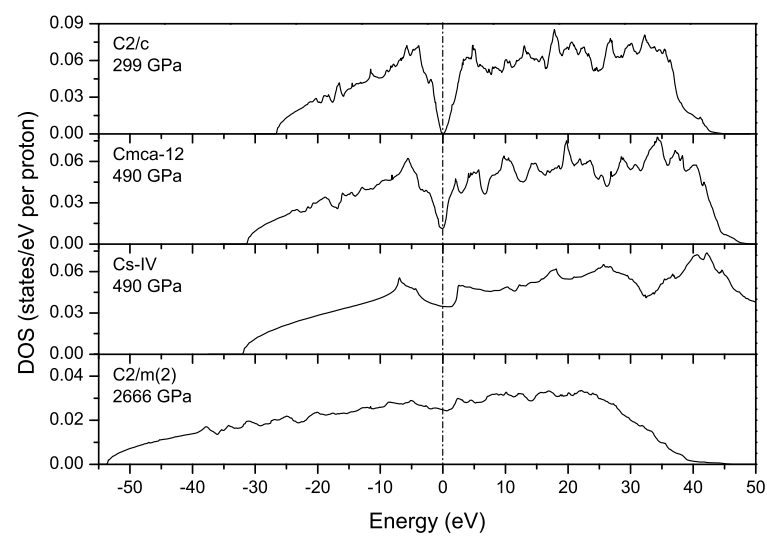

FIG. 5. Electronic density of states of dense hydrogen at high pressures. The dash-dotted vertical line indicates the Fermi level. Notice that $C 2 / m(2)$ at a pressure of $2.7 \mathrm{TPa}$ has typical features of simple metals.

gap by band overlapping at the Fermi level. On the other hand, Cs-IV and $C 2 / m(2)$ show typical characteristics of simple metals in their DOS. Especially, the DOS of $C 2 / m(2)$ has already been dispersed greatly by compression and becomes flat and featureless over a wide range of energy. Other phases of dense hydrogen at ultra-high pressures are similar and it is unnecessary to discuss them separately.

\section{B. Effects of zero-point motion of protons}

\section{Harmonic ZPE and its failure}

The contribution of zero-point vibrations of protons to energy and enthalpy can be taken into account within quasi-harmonic approximation. Since in a static lattice approximation our calculated groundstates and the relative stable order of high pressure phases of dense hydrogen are almost the same as that reported in Ref.19, and they had performed a detailed analysis of the zero point energy (ZPE) in harmonic approximation, thus it is not necessary to repeat the discussion. Here we only illustrate the magnitude and the possible consequence of harmonic ZPE using meta-stable multi-atomic molecular phases as examples. As Fig,6 shows, inclusion of harmonic ZPE slightly changes the relative stability of these phases. The magnitude of $\mathrm{ZPE}$ is about $0.32 \mathrm{eV}$ per proton at $1 \mathrm{TPa}$, and increases to $0.42 \mathrm{eV}$ per proton at $2 \mathrm{TPa}$. For highly compressed atomic phases of hydrogen, this treatment is inappropriate 


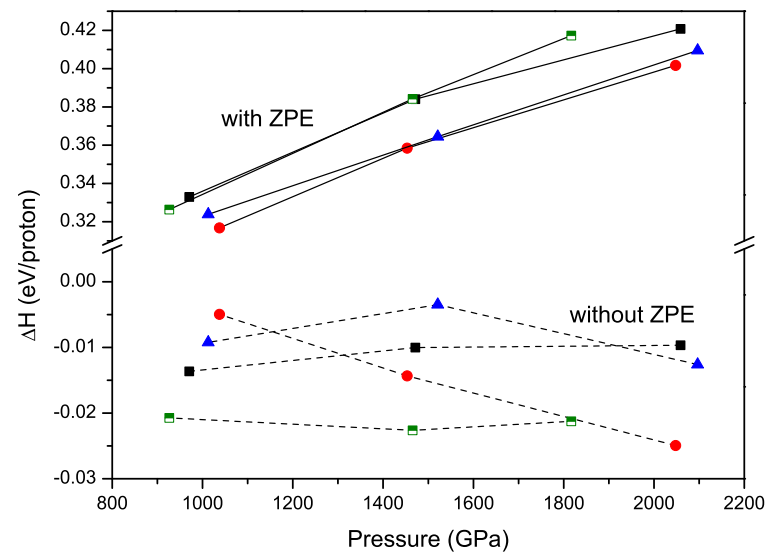

FIG. 6. (color online) Change of the relative stability of meta-stable dense hydrogen due to contribution from harmonic ZPE, where the solid (dased) lines indicate with (without) ZPE, $\Delta H$ stands for the enthalpy difference with respect to a predesignated reference state, and the filled circles, squares, triangles, and half-filled squares denote $C 2 / m(2), C 2 / m(1), \beta-H g$, and Pmmn phases, respectively.

because of the strong anharmonic effects. $\underline{43-46}$ However, for multi-atomic molecular phases, the anharmonic effects are also remarkable and the shape of the potential well around the equilibrium position of each proton is far from being a quadratic form (see below), which undermines the justification for harmonic approximation. In this sense the relative stability of dense hydrogen cannot be faithfully determined by harmonic ZPE, because the enthalpy difference between these phases is too small, and at the same time it is almost impossible for the harmonic approximation to have a precision of within several percents in the case of dense hydrogen.

On the other hand, most of the energy barriers that separate different phases of dense hydrogen are much smaller than the magnitude of harmonic ZPE. In this situation, the classical notation of structural phase might be ill-defined, because it is easy for the system to overcome the barriers and travel freely from one phase into another driven by ZP motions. Such kind of quantum fluctuation between structures invalidates not only harmonic approximation of lattice dynamics, but also some restricted quantum Monte Carlo (QMC) treatments. $\underline{43}, \underline{44}$ To tackle this problem quantitatively, a full quantum treatment of protons on the same footing as electrons is required, $\stackrel{47}{48}$ which however, is difficult within the DFT framework based on Born-Oppenheimer approximation. 


\section{Anharmonic zero-point motion}

Although in DFT it is difficult to carry out a quantitative analysis of the anharmonic ZP effects if nuclei are treated as classical particles, an insightful perception of the quantum motion of protons still can be obtained by inspecting the energy surface or landscape closely. Figure 7 shows a series of section of the energy surface that cut along a transition path from FCC to $\beta$-Hg at different densities. That is to say, changing the lattice length $c$ of the $\beta$-Hg structure while adjusting the $a$ and $b$ accordingly at a fixed density with $r_{s}$ equaling $1.12(\sim 1 \mathrm{TPa}), 1.04(\sim 1.5 \mathrm{TPa})$, and $1.01(\sim 2 \mathrm{TPa})$, respectively. Here the dimensionless parameter $r_{s}$ is defined as the radius of a sphere which encloses on the average one electron in a unit of the Bohr radius $\underline{\underline{20}-\underline{22}}$ A value of $r_{s}=1$ corresponds to a physical compression ratio of about 30 for hydrogen. The energy surface obtained in this way is approximation free, except those that already introduced in the standard DFT formalism. Particles move on these surfaces at $0 \mathrm{~K}$ as zero point motions.

It is easy to find from these surfaces that the harmonic approximation breaks down completely for monatomic phases. There is even no potential well can be defined between BCC and FCC (and between BCC and $\beta$ - $\mathrm{Hg}$ as well). The flatness of the energy surface unveils an important origination of the unusually large anharmonic effects in monatomic phases of hydrogen observed in QMC calculations (in addition to the light mass of protons): $: \underline{43} \underline{46}$ having such a flat energy surface, the crystal might melt even at zero Kelvin.

On the other hand, the harmonic ZP root mean square displacement (rMSD) gives a typical value of $0.2 \AA$ for $\beta-\mathrm{Hg}$ phase. $\underline{\underline{49}}$ It characterizes a typical ZP motion size for protons in a chained molecular phase. Making use of this value and the potential well width given in Fig.7, we estimated that at a pressure of $1 \mathrm{TPa}$ the ZP motions of protons are confined within the potential well. But with increasing of the pressure, ZP vibrational size becomes comparable with the potential well width at $1.5 \mathrm{TPa}$, and exceeds the latter at $2 \mathrm{TPa}$. Namely, beyond that pressure $\beta$-Hg might merge into $\mathrm{FCC}$ and $\mathrm{BCC}$ phases driven by ZP motions. This picture can be understood by analogy with an effective particle that moves on a similar energy surface (a half-infinite potential well), as shown in the inset of Fig.7. In that case a bound state (i.e., a well-defined crystal) exists only when $8 M V x^{2} \geq \pi^{2} \hbar^{2}$ where $x(V)$ is the

potential width(depth) and $M$ is the particle mass. $\underline{50}$ When no bound state is available, the particle moves around freely, but a phase cancellation between the incident and reflected 


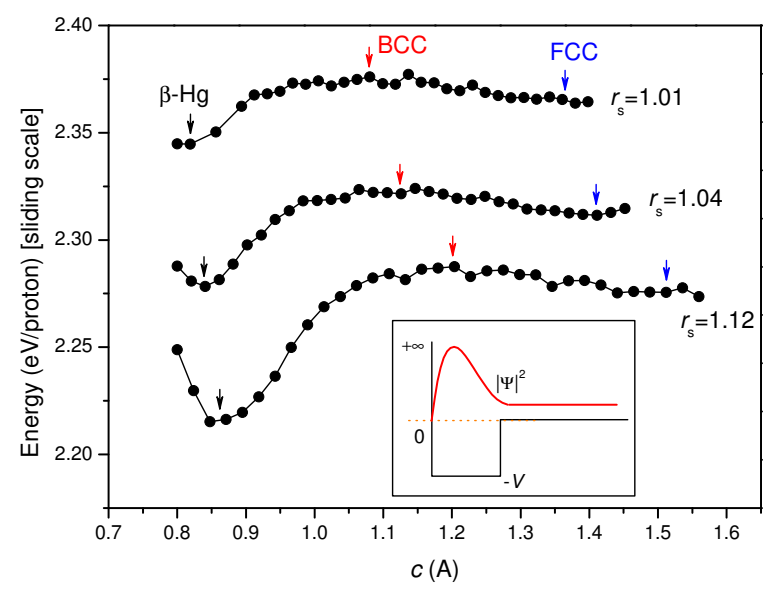

FIG. 7. (color online) Energy variation with the change of the $c$ axial length of the $\beta$-Hg structure, which passes through FCC, BCC, and $\beta$-Hg successively. Inset: a simplified half-infinite potential well that models the energy surface, as well as the corresponding probability density profile of an effective particle moving in it.

waves make it have a relative high probability within the potential well, as the probability density profile in the inset of Fig, 7 illustrates.

Alternatively, this phenomenon can also be understood intuitively via the path integral formalism of quantum statistics theory. The partition function of a system consisting of distinguishable particles (here hydrogen atoms) can be written as ${ }^{51}$

$$
Z=\int \rho(R, R ; \beta) d R
$$

with the diagonal density matrix given by

$$
\rho(R, R ; \beta)=\rho^{0}(R, R ; \beta)\left\langle\exp \left[-\int_{0}^{\beta} V\left(R_{t}\right) d t\right]\right\rangle_{B W} .
$$

Here $\rho^{0}$ is the free particle density matrix, $\beta$ denotes $\left(k_{B} T\right)^{-1}$ where $T$ is the temperature, and the potential contribution (the \langle\rangle$_{B W}$ term) is given by averaging over Brownian motions along closed paths that weighted by potential energy. The total energy with ZP contribution included is thus

$$
E_{0}=-\lim _{\beta \rightarrow \infty} \frac{\partial \ln Z}{\partial \beta} .
$$

In cases where structural phases are separated by high enough barriers, the energy $E_{0}$ is locally defined. Namely, all Brownian motions in Eq.(2) are effectively confined to a limited phase space which characterizes the structure, and therefore one can compare the value of $E_{0}$ to determine the relative stability between different phases. 
However, when the energy barrier is low or even no barrier at all, $E_{0}$ of one phase contains contribution of the Brownian paths winding across other phases, and the definition of the energy of that phase becomes meaningless. In this situation one could instead analyze the probability for a Brownian motion to fall into a specific region in the phase space. Taking $\beta$-Hg for example, the probability for this phase to appear is given by

$$
\eta=\frac{\int_{\Omega_{m}} \rho(R, R ; \beta) d R}{\int_{\Omega} \rho(R, R ; \beta) d R},
$$

where $\Omega_{m}$ is the domain of the phase space defined by the structure of $\beta$-Hg, i.e., an analogue of the width of the potential trap as shown in Fig:7, and $\Omega$ is the whole phase space domain that is accessible to all Brownian motions. Because the potential well is attractive, the paths that belong to/or pass through the potential well $\left(\Omega_{m}\right)$ always have a higher weight than others. Therefore $\eta$ has a non-zero value at low enough temperatures. With the temperature decreases further, $\Omega$ gradually shrinks backwards to $\Omega_{m}$. At zero Kelvin, if $\Omega$ reduces to $\Omega_{m}$ exactly, then the phase of $\beta-\mathrm{Hg}$ is a well defined classical structure. Otherwise there are quantum fluctuations and $\beta-\mathrm{Hg}$ exists only instantaneously with a probability of $\eta$. Note this kind of quantum behavior that blurs the structural boundary of phases had been noticed in path integral simulations, $\stackrel{45,46}{\underline{46}}$ which treated the quantum nature of protons. Here we considered only meta-stable phases, similar argument (though not identical) can be applied to the groundstate Cs-IV and its distortions.

\section{Energy barrier of phase transition}

From above discussion, we knew that the shape of the energy surface and the barrier that separates different phases are crucial content to understand the high pressure behavior of dense hydrogen comprehensively. The transition path shown in Fig:7 is a special case, which reveals that no energy barrier presents between chained $\beta$-Hg and cubic FCC or BCC phases. Furthermore, the potential well around $\beta$-Hg becomes shallower and shallower with increasing pressure, indicating the enhancement of the stability of high-symmetric phases and the weakening of the tendency to form low coordinated structures. It should be noted that this path is along a preassigned route. We can do this because the transition path is

fixed completely by symmetry and structure analysis. However, this is not always the case, and a general technique such as NEB has to be employed in order to map out the transition 


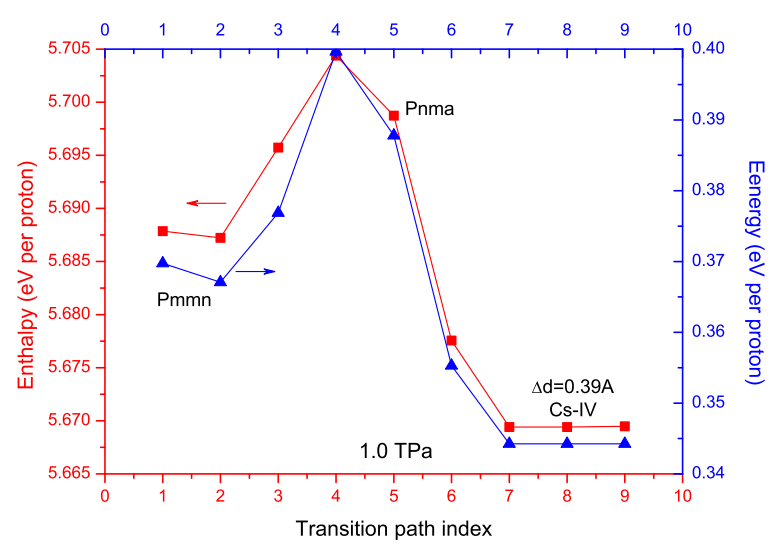

FIG. 8. (color online) Energy and enthalpy variations along the phase transition path from Pmmn to Cs-IV at $1 \mathrm{TPa}$. Note that Cs-IV has a freedom to drift far away from its ideal position.

path.

Figure 8 illustrates the transition path from meta-stable Pmmn to the groundstate Cs-IV phase at $1 \mathrm{TPa}$ calculated with NEB. Both variations of energy and enthalpy are shown. A distinct barrier $(0.035 \mathrm{eV} /$ proton in enthalpy and $0.055 \mathrm{eV} /$ proton in energy from the Cs-IV side) was clearly obtained. From the energy variation, we can see that both of the end phases have distortions with a small energy change. In particular, the groundstate Cs-IV has various variants which possess almost the same energy or enthalpy. The average drift distance of atoms between these variants can be as large as $\triangle d=0.39 \AA$, compared with the NN distance of $0.92 \AA$ in this structure. This implies that it might be quite common for broad and flat basins to present at some locally stable phases (even the groundstate) of dense hydrogen, which was never noticed before. Furthermore, the flatness of the basins implies that the ZPE should be much smaller than that predicted by harmonic approximation. In addition, since an enthalpy barrier of $0.035 \mathrm{eV} /$ proton from Cs-IV to the first meta-stable phase Pmmn corresponds to a temperature scale of $400 \mathrm{~K}$, it is highly possible that dense hydrogen is in a solid state at this pressure range at room temperature. Of course, considering that Cs-IV itself has many variants with negligible energy change, it is also possible that it melts locally driven by ZP motions. If this is the case, then a liquid-liquid transition could be expected when temperature is increased.

Being analogous to Fig:7, a well-defined barrier is absent between the meta-stable Pmmn and $C 2 / m(2)$ at $2.8 \mathrm{TPa}$, as shown in Fig.9. There are three noticeable characteristics presented in this path: (i) a more stable $R \overline{3} m$ phase manifests itself in the transition path; 


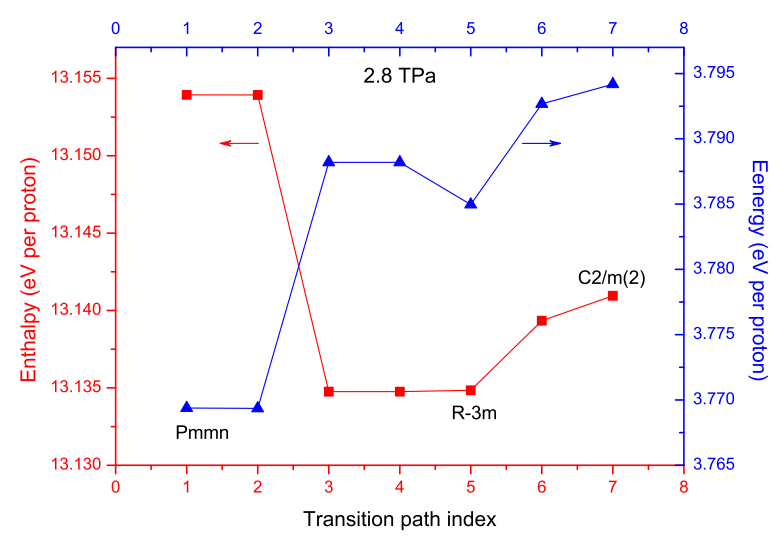

FIG. 9. (color online) Energy and enthalpy variations along the phase transition path from Pmmn to $C 2 / m(2)$ at $2.8 \mathrm{TPa}$. Note that the energy variation does not comply with that of enthalpy, and a more stable phase of $R \overline{3} m$ is unveiled.

(ii) a flat energy (or enthalpy) surface appears again; and (iii) the energy variation is inverse to that of enthalpy. The first point shows that it is possible to find out more stable structures by investigating the transition path between high-lying meta-stable phases. This is also helpful for understanding the physical mechanism of structure stability. The second point reflects the frustration of different competing factors, and implies that it is ineffective to optimize the structure by conventional relaxation algorithms because the forces become too small to evolve the geometry within these flat regions. The final point clearly implies that $C 2 / m(2)$ is stabilized by the term of $P V$, and should have great imaginary phonon frequencies in harmonic approximation because the lattice dynamical matrix is evaluated on the energy surface. However, such imaginary modes do not necessarily mean that the phase is locally unstable in thermodynamics if one takes the $P V$ contribution into account.

It is interesting to investigate the transition path from diatomic molecular $\mathrm{Cmca}-12$ to atomic Cs-IV at the dissociation pressure of $495 \mathrm{GPa}$. As Fig.10 shows, a high enthalpy barrier of $\triangle H=0.038 \mathrm{eV} /$ proton presents. This will introduce hysteresis and lift the apparent dissociation pressure at low temperatures. Since the volume difference per atom between $C m c a-12$ and $\mathrm{Cs}-\mathrm{IV}$ is about $\triangle V=0.03 \AA^{3}$, the possible pressure increase is thus $\triangle P=\triangle H / \triangle V \approx 200 \mathrm{GPa}$ to the first order of correction. ${ }^{23}$ That is to say, the DFT predicted dissociation pressure of diatomic hydrogen should be at about $700 \mathrm{GPa}$ if taking the kinetic effect of enthalpy barrier into account. Other factors that might also have some impacts include: (i) ZP motion of protons, which favors atomic phases and therefore should 


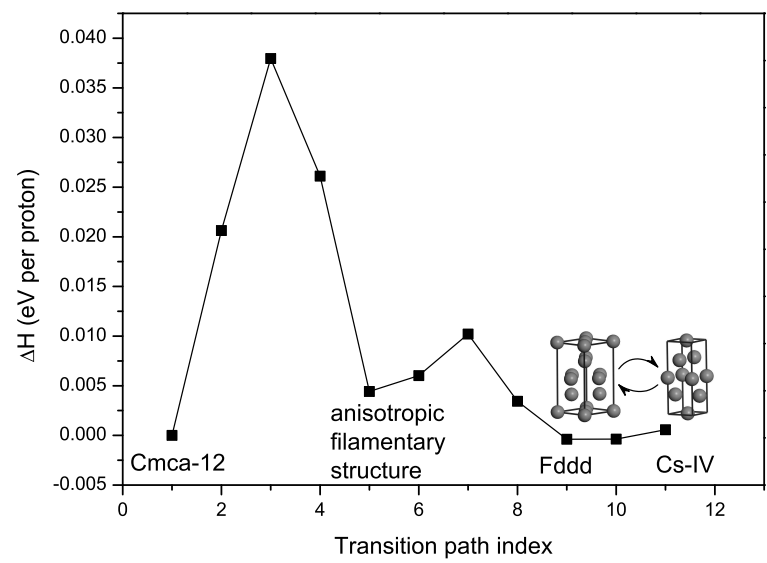

FIG. 10. Enthalpy variation of dense hydrogen along the transition path from diatomic molecular Cmca-12 to atomic Cs-IV phase at $495 \mathrm{GPa}$, where a low-symmetric filamentary phase and Fddd phase (a distorted diamond structure) were observed.

decrease the dissociation pressure,$\frac{19}{43,44,52}$ but its precise value is hard to evaluate at present; (ii) intrinsic DFT error which favors homogeneous distribution of electronic density, thus also underestimates the dissociation pressure about $50 \mathrm{GPa} ; \stackrel{5}{-5}$ (iii) other transition path with lower enthalpy barrier, we cannot exclude this possibility completely because NEB is in fact a local optimization algorithm, and it explores a limited phase space and thus depends on the initial guess of the transition path in some degree. Nevertheless, after taking all of these factors into account the dissociation pressure should be less than $750 \mathrm{GPa}$, or $550 \mathrm{GPa}$ if no any hysteresis effect presents.

Another interesting phenomenon unveiled in Fig 10 is that there is no energy barrier between the degenerate groundstates of Cs-IV and Fddd. In other words, dense hydrogen fluctuates between these two structures driven by ZP motions. Note that although Fddd can be viewed as a distortion of Cs-IV structure, from a crystallographic point of view, it is in fact an orthorhombic variant of the diamond phase. Namely, the quantum structural fluctuation in the groundstate of dense hydrogen is much more drastic than what Fig 8 has been implied. Also an anisotropic low-symmetry filamentary phase manifests itself on the transition path, which is very similar to the previously proposed low-symmetric structures of dense hydrogen $\underline{45,46,53-55}$ It is separated from other phases by an energy barrier and might be meta-stable. 


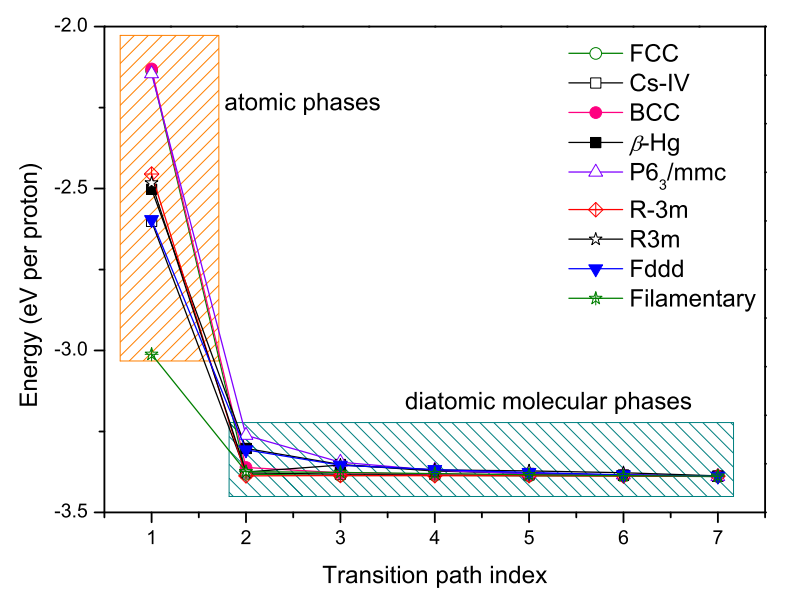

FIG. 11. (color online) Energy variation of a series of dense hydrogen phases along the respective transition path to a diatomic molecular structure at zero pressure. No energy barrier can be detected.

\section{Stability of dense hydrogen at zero pressure}

The stability of atomic phases of dense hydrogen at zero pressure is intriguing because

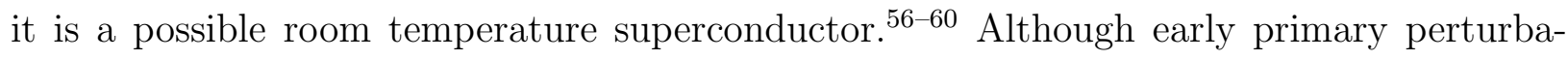
tion calculations on the structural energy of hydrogen within a static-lattice approximation with the effective electron-ion interaction expanded to fourth order suggested that a highly anisotropic structure might be stable, $, \underline{53}, \underline{54}$ later more careful analyses dismissed this proposal and found that isotropic phases are more favored. $\underline{43,44,52}$ The energetic stability of these recently proposed new groundstates and low-lying meta-stable phases ${ }^{16,17,19}$ at $0 \mathrm{GPa}$, however, has not been studied yet. Namely, whether these high pressure phases of hydrogen is quenchable or not is still unknown.

To answer this question, we investigated the transition path and the associated energy barrier of these dense phases to diatomic molecular structures at $0 \mathrm{GPa}$ with NEB calculations. The initial dense structures were prepared by carefully relaxing the configurations from high pressures with constraints applied. The corresponding diatomic molecular phases were then produced by distorting and relaxing the respective dense structures. Typical results are illustrated in Fig.11. The conclusion is that all of the low-lying states of dense hydrogen known so far, including isotropic atomic phases and low coordinated tri-atomic or chained molecular phases, have no transition barriers can be detected along the transformation path. In particular, the exotic filamentary structure observed in Fig 10 has no energy 


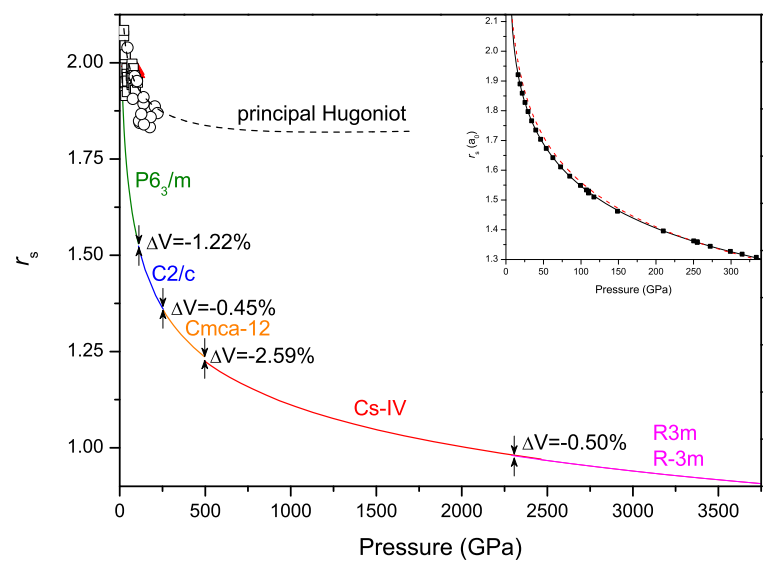

FIG. 12. Calculated equation of state at $0 \mathrm{~K}$ of dense hydrogen up to $3.5 \mathrm{TPa}$ compared with the extrapolation of the principal Hugoniot. Inset shows the comparison with the Vinet EOS (dashed line) that fitted to experimental data up to $119 \mathrm{GPa}$ at low pressures.

barrier, too. That is to say, there is nothing that can prevent these phases from spontaneously decaying to diatomic molecular structures. This fact that dense hydrogen might be unquenchable is a direct consequence of the strong tendency of hydrogen to pair at low pressures (Pmmn and $C 2 / m$ are also unstable at $0 \mathrm{GPa}$, which didnot include in Fig.11). This result is obtained with DFT in PBE approximation. This exchange-correlation energy functional constructed on a local or semi-local approximation to the homogeneous electron gas value overestimates the stability of a metallic phase slightly, but cannot eliminate the whole energy barrier completely if there were one. Also, an insightful analysis of the intrinsic error in DFT $\underline{61,62}$ implies that it seems unlikely that an additional energy barrier can be predicted by an exact theory of DFT when no any barrier can be detected with the current version of DFT. That is to say, even in a level of the exact many-body quantum theory, dense hydrogen might still be unquenchable for these already known structures.

\section{E. Equation of state at zero Kelvin}

Equation of state (EOS: the pressure-volume relation here) is an important content to understand the response of a material to compressions, which reflects not only the phase transitions driven by pressure, but also the evolution of interactions with the change of coordination environment. In experiment, x-ray diffraction measurements of the structure of single-crystal molecular hydrogen had been performed at pressures up to $109 \mathrm{GPa}$ for $\mathrm{H}_{2}$ 
and $119 \mathrm{GPa}$ for $\mathrm{D}_{2} \cdot \stackrel{24}{ }$ From these measurements a high-pressure EOS was deduced, 24 which is significantly more compressible than that early constrained from lower-pressure data to

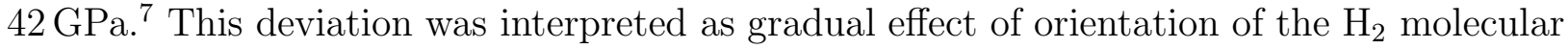
axis within phase I. ${ }^{24}$ At higher pressures, hydrogen transforms into atomic Cs-IV phase, where both interatomic interactions and crystal structure are changed. Therefore there is enough reason to suspect that this experimental EOS cannot be extrapolated to higher pressure range.

With the information of the calculated groundstate structures, the whole pressure-volume curve of dense hydrogen at zero Kelvin was calculated up to 3.5 TPa with DFT. Figure 12 shows this curve by comparing with the extrapolation of the principal Hugoniot. It is worthwhile to note that although there is a little difference between $R 3 m$ and $R \overline{3} m$ in the enthalpy and it is hard to determine which one is the true groundstate at ultra-high pressures, both phases have an almost identical $P-V$ relation. Similarly, $F d d d$ has the same variation of density with pressure as that of Cs-IV and its distortions, and thus didnot include in Fig.12. Along the groundstate line, the volume collapses due to phase transitions are small, it is about $2.59 \%$ when dissociates to Cs-IV phase and $1.22 \%$ between $P 6_{3} / m$ and $C 2 / c$. In other transitions it is just about $0.5 \%$.

The experimental data can be fitted to a Vinet function, ${ }^{24}$ which is overall in good agreement with our DFT calculated data below $300 \mathrm{GPa}$, as the inset of Fig.12 shows. However, there is a subtle discrepancy: the DFT overestimates the compressibility slightly when between 50 and $150 \mathrm{GPa}$. This might be due to the fact that we didnot treat the quantum rotation of $\mathrm{H}_{2}$ molecules explicitly. This degree of freedom of motion should contribute energy and thus increase the internal pressure accordingly. At higher pressures, however, extrapolation of the fitted Vinet function overestimates the compressibility significantly. As Fig 13 illustrates, both of the Vinet function that fitted to original experimental data (Vinet $300 \mathrm{~K}$ ) and that fitted to data reduced to $0 \mathrm{~K}$ without ZP contribution (Vinet $0 \mathrm{~K}$ ) deviate from the DFT results when beyond $500 \mathrm{GPa}$, and the difference can reach as high as $36 \%$ at a density of $r_{s}=0.9$. Note that the curves of "Vinet $300 \mathrm{~K}$ " and "Vinet $0 \mathrm{~K}$ " are almost identical within the studied pressure range, showing that both the ZP pressure and thermal pressure are relatively small in comparison. Furthermore, since in DFT calculations we didnot take ZP contribution into account and usually ZP motion contributes a positive pressure and thus should reduce the compressibility, the DFT EOS shown in Fig.13 is thus 


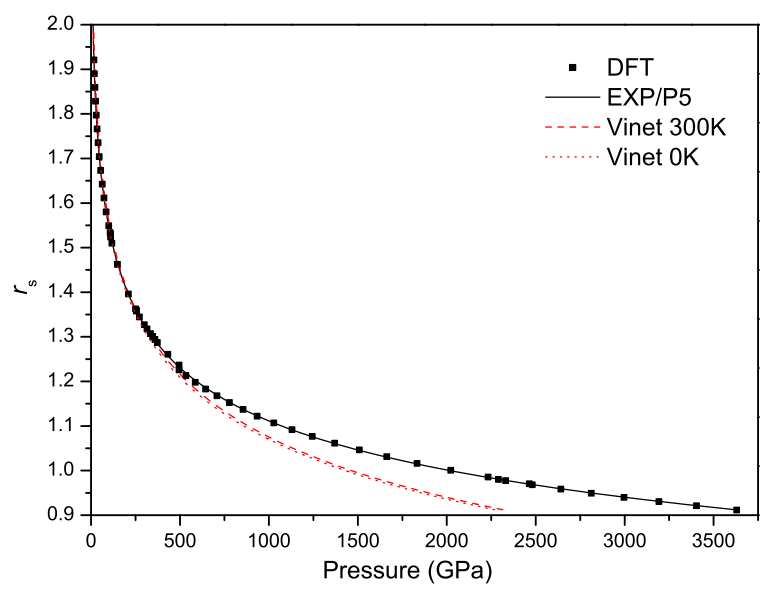

FIG. 13. Comparison of the calculated pressure-volume curve with the Vinet EOS models that fitted to low-pressure data and a proposed EXP/P5 function within a pressure range up to $3.5 \mathrm{TPa}$.

an upper estimate of the compressibility of dense hydrogen, and any model with a higher compressibility (e.g., the fitted Vinet EOS) is not allowed in physics.

If ignored the small volume collapses at the phase transition points, the pressure-volume relation of the groundstates of dense hydrogen at $0 \mathrm{~K}$ calculated by DFT can be fitted excellently by a function of

$$
P=\prod_{n=0}^{5} 10^{A_{n} r_{s}^{n}} .
$$

We denote it as EXP/P5. When the pressure $P$ is in a unit of GPa, the parameters are as follows: $A_{0}=1.0683, A_{1}=19.1824, A_{2}=-36.3776, A_{3}=28.5165, A_{4}=-10.6068$, and $A_{5}=1.5224$. It should be noted that $\mathrm{EXP} / \mathrm{P} 5$ function not only faithfully represents the overall variation of the EOS of dense hydrogen over a broad range of pressure up to 3.5 TPa, it also reproduces the low pressure data accurately, as demonstrated in the inset of Fig, 12 . With these properties, this function should have a good applicability for extrapolating the EOS of hydrogen to higher pressures beyond several TPa.

\section{CONCLUSION}

High pressure phases of dense hydrogen have been extensively searched up to 24 atoms per unit cell and 3.5 TPa in pressure with density functional theory calculations. The results confirmed the previous conclusion that diatomic hydrogen dissociates into atomic Cs-IV at $495 \mathrm{GPa}$ and then transforms to $R 3 m$ or $R \overline{3} m$ at about $2.3 \mathrm{TPa}$. Exotic high pressure 
behaviors were also discovered that show dense hydrogen having numerous isomorphs with comparable enthalpy, and frustrated competitions among them lead to broad and flat basins on the energy surface. In particular, calculations showed that there is even no energy barrier to separate crystallographic different phases in some cases. Furthermore, the atomic groundstate Cs-IV has a freedom to distort and is degenerate with orthorhombic Fddd, which fluctuates forth and back to Cs-IV driven by ZP motions of protons.

Within a wide range of pressure beyond $500 \mathrm{GPa}$, the first meta-stable structure is a triatomic Pmmn, therefore the stability of the closely related multi-atomic molecular phases was also analyzed. Electronic structure calculations indicated that they are metallic in nature, but with weak covalent bonds presented. The occurrence of these meta-stable phases is a direct consequence of the competition between the tendency to overlap the electron orbitals among neighboring atoms and the pressure-induced dissociation of molecular hydrogen. The structural relationship between these exotic phases was investigated for a better understanding of the structural behavior of dense hydrogen.

In addition to static-lattice calculations, ZP vibrations of lattice were also computed in the harmonic approximation and the magnitude of its contribution was estimated. Inspection of the energy surface (via transition path) dismissed the validity of this level approximation, and the anharmonicity of ZP motions was demonstrated by studying the variation of the energy surface with pressure, which not only reveals the weakening of the tendency to form covalent bonds under compression, but also illustrates the importance of quantum fluctuations in structure of dense hydrogen at high pressures.

The general transition path and the associated energy barrier between different phases were calculated with NEB technique, which suggested that the dissociation pressure of diatomic molecular hydrogen might be deferred to at about $750 \mathrm{GPa}$ due to a hysteresis effect of the energy barrier at low temperatures. The enthalpy barrier between the groundstate Cs-IV and the first meta-stable Pmmn phase at $1 \mathrm{TPa}$ implies atomic hydrogen might be in a solid state at zero Kelvin, but it depends on the exact contribution of anharmonic ZP vibrations of protons. The calculated transition path also suggested that there are phases in dense hydrogen that are stabilized purely by $P V$ term, which is unusual in the common sense. The meta-stability of dense phases of hydrogen at zero pressure were extensively studied. No energy barrier was detected between them and the diatomic molecular phase, implying dense hydrogen might be unquenchable. 
The equation of state (i.e., the pressure-volume relation) of dense hydrogen up to $3.5 \mathrm{TPa}$ was investigated. A new EOS function, namely EXP/P5, was proposed, which can represent the calculated DFT data over the whole studied pressure range excellently. Since no thermal pressure and ZP contribution were included, this EOS is an upper estimation of the true compressibility, provided that the current understanding of the groundstate structure of dense hydrogen is correct. At low pressures, this EOS is in good agreement with the experimental data measured by single crystal x-ray technique. Extrapolating the Vinet EOS model that fitted to these measured low-pressure data, however, drastically overestimates the compressibility when beyond $500 \mathrm{GPa}$, due to inappropriate counting of the interatomic repulsion under extreme compressions in this model. By the way, as isotopes of hydrogen, deuterium and tritium have almost the same electronic behavior. The main difference is that they have a heavier ionic mass, and thus the ZP effects should be less significant. But the overall picture of the physics is the same.

1 T. Guillot, Science 286, 72 (1999).

2 T. Guillot, Planet. Space Sci. 47, 1183 (1999).

3 S. A. Bonev, E. Schwegler, T. Ogitsu, and G. Galli, Nature 431, 669 (2004).

4 S. Deemyad and I. F. Silvera, Phys. Rev. Lett. 100, 155701 (2008).

5 M. A. Morales, C. Pierleoni, E. Schwegler, and D. M. Ceperley, Proc. Natl. Acad. Sci. U.S.A. 107, 12799 (2010).

6 W. Lorenzen, B. Holst, and R. Redmer, Phys. Rev. B 82, 195107 (2010).

7 H. K. Mao and R. J. Hemley, Rev. Mod. Phys. 66, 671 (1994) (and references therein).

8 J. H. Eggert, F. Moshary, W. J. Evans, H. E. Lorenzana, K. A. Goettel, I. F. Silvera, and W. C. Moss, Phys. Rev. Lett. 66, 193 (1991).

9 C. Narayana, H. Luo, J. Orloff, and A. L. Ruoff, Nature 393, 46 (1998).

10 P. Loubeyre, F. Occelli, and R. Le Toullec, Nature 416, 613 (2002).

11 U. Bafile, F. Becherini, D. Colognesi, and M. Zoppi, Phys. Rev. B 77, 224302 (2008).

12 E. Wigner and H. B. Huntington, J. Chem. Phys. 3, 764 (1935).

13 C. Friedli and N. W. Ashcroft, Phys. Rev. B 16, 662 (1977).

14 T. W. Barbee, III, A. Garcia, M. L. Cohen, and J. L. Martins, Phys. Rev. Lett. 62, 1150 (1989). 
15 H. Chacham and S. G. Louie, Phys. Rev. Lett. 66, 64 (1991).

16 K. A. Johnson and N. W. Ashcroft, Nature 403, 632 (2000).

17 C. J. Pickard and R. J. Needs, Nature Phys. 3, 473 (2007).

18 J. S. Tse, D. D. Klug, Y. S. Yao, Y. L. Page, and J. R. Rodgers, Solid State Commun. 145, 5 (2008).

19 J. M. McMahon and D. M. Ceperley, Phys. Rev. Lett. 106, 165302 (2011).

20 E. Wigner, Phys. Rev. 46, 1002 (1934).

21 W. J. Carr, Jr., Phys. Rev. 122, 1437 (1961).

22 M. D. Jones and D. M. Ceperley, Phys. Rev. Lett. 76, 4572 (1996).

23 H. Y. Geng, Y. Chen, Y. Kaneta, and M. Kinoshita, Phys. Rev. B 75, 054111 (2007).

24 P. Loubeyre, R. LeToullec, D. Hausermann, M. Hanfland, R. J. Hemley, H. K. Mao, and L. W. Finger, Nature 383, 702 (1996).

25 C. J. Pickard and R. J. Needs, Phys. Rev. Lett. 97, 045504 (2006).

26 B. Rousseau, Y. Xie, Y. Ma, and A. Bergara, Eur. Phys. J. B 81, 1 (2011).

27 J. Lv, Y. Wang, L. Zhu, and Y. Ma, Phys. Rev. Lett. 106, 015503 (2011).

28 Y. Yao, J. S. Tse, and D. D. Klug, Phys. Rev. Lett. 102, 115503 (2009).

29 Y. Wang, J. Lv, L. Zhu, and Y. Ma, Phys. Rev. B 82, 094116 (2010).

30 G. Kresse and J. Furthmüller, Phys. Rev. B 54, 11169 (1996).

31 P. E. Blöchl, Phys. Rev. B 50, 17953 (1994).

32 G. Kresse and D. Joubert, Phys. Rev. B 59, 1758 (1999).

33 J. P. Perdew, K. Burke, and M. Ernzerhof, Phys. Rev. Lett. 77, 3865 (1996).

34 M. I. McMahon and R. J. Nelmes, Chem. Soc. Rev. 35, 943 (2006).

35 G. Mills, H. Jonsson, and G. K. Schenter, Surf. Sci. 324, 305 (1995).

36 D. Alfè, Comp. Phys. Comm. 180, 2622 (2009).

37 This state does not correspond to a physical one. It is artificially defined by the enthalpy only for the purpose to unveil the subtle enthalpy difference among competing phases.

38 R. E. Peierls, Quantum Theory of Solids (Oxford University Press, London, 1955).

39 A. D. Becke and K. E. Edgecombe, J. Chem. Phys. 92, 5397 (1990).

40 A. Savin, J. Mol. Struct.: THEOCHEM 727, 127 (2005).

41 M. Marques, M. I. McMahon, E. Gregoryanz, M. Hanfland, C. L. Guillaume, C. J. Pickard, G. J. Ackland, and R. J. Nelmes, Phys. Rev. Lett. 106, 095502 (2011). 
42 Y. Ma, M. Eremets, A. Oganov, Y. Xie, I. Trojan, S. Medvedev, A. Lyakhov, M. Valle, and V. Prakapenka, Nature (London) 458, 182 (2009).

43 V. Natoli, R. M. Martin, and D. M. Ceperley, Phys. Rev. Lett. 70, 1952 (1993).

44 V. Natoli, R. M. Martin, and D. M. Ceperley, Phys. Rev. Lett. 74, 1601 (1995).

45 S. Biermann, D. Hohl, and D. Marx, Solid State Commun. 108, 337 (1998).

46 S. Biermann, D. Hohl, and D. Marx, J. Low Temp. Phys. 110, 97 (1998).

47 K. Johnson and N. W. Ashcroft, J. Phys.: Condens. Matter 10, 11135 (1998).

48 N. W. Ashcroft, J. Phys.: Condens. Matter 12, A129 (2000).

49 This value is comparable with the root-mean-square radius of gyration of a nucleus in ab initio path integral simulations that have taken the quantum effects of protons into account fully, see Refs, 45 and 46.

50 L. D. Landau and E. M. Lifshitz, Quantum Mechanics (Non-relativistic Theory), 3rd edition (Elsevier, Singapore, 1965).

51 D. M. Ceperley, Rev. Mod. Phys. 67, 279 (1995).

52 D. M. Straus and N. W. Ashcroft, Phys. Rev. Lett. 38, 415 (1977).

53 E. G. Brovman, Y. Kagan, and A. Kholas, Zh. Eksp. Theor. Fiz. 61, 2429 (1971) [Sov. Phys. JETP 34, 1300 (1972)].

54 E. G. Brovman, Y. Kagan, and A. Kholas, Zh. Eksp. Theor. Fiz. 62, 1492 (1972) [Sov. Phys. JETP 35, 783 (1972)].

55 E. Kaxiras, J. Broughton, and R. J. Hemely, Phys. Rev. Lett. 67, 1138 (1991).

56 N. W. Ashcroft, Phys. Rev. Lett. 21, 1748 (1968).

57 L. J. Zhang, Y. L. Niu, Q. Li, T. Cui, Y. Wang, Y. M. Ma, Z. He, and G. T. Zou, Solid State Commun. 141, 610 (2007).

58 E. G. Maksimov and D. Y. Savrasov, Solid State Commun. 119, 569 (2001).

59 R. Szczesniaka and M. W. Jarosik, Solid State Commun. 149, 2053 (2009).

60 J. M. McMahon and D. M. Ceperley, Phys. Rev. B 84, 144515 (2011).

61 R. O. Jones and O. Gunnarsson, Rev. Mod. Phys. 61, 689 (1989).

62 A. J. Cohen, P. Mori-Sanchez, and W. Yang, Science 321, 792 (2008).

63 A. Savin, C. J. Umrigar, and X. Gonze, Chem. Phys. Lett. 288, 391 (1998).

64 M. A. Morales, C. Pierleoni, and D. M. Ceperley, Phys. Rev. E 81, 021202 (2010).

65 M. Städele and R. M. Martin, Phys. Rev. Lett. 84, 6070 (2000). 
66 P. Blaha, K. Schwarz, G. K. H. Madsen, D. Kvasnicka, and J. Luitz, WIEN2k, An Augmented Plane Wave + Local Orbitals Program for Calculating Crystal Properties (Karlheinz Schwarz, Techn. Universität Wien, Austria), 2001. ISBN 3-9501031-1-2

\section{Appendix A: Justification to the density functional theory}

The sensitivity of the calculated results to the choice of exchange-correlation density functional had been checked in Ref.17. We repeated the checking process and obtained similar conclusions. Furthermore, the validity of the density functional theory to ultrahigh pressure physics can be understood easily. We know that the apparent failure of all local density approximation (LDA) based functionals relates directly to an abrupt variation of charge density. Hydrostatic compression reduces interatomic distance, which increases interactions between electrons and nearby nuclei. Covalent bonds are thus weakened and electronic wave-function spreads out due to environmental changes in atomic coordination usually. The direct consequence is thus a more smooth spatial distribution of the charge density, and a better performance of LDA-based functionals with an increase of the pressure (note that the peculiar phenomenon of pressure-induced localization of electrons observed in lithium $27, \underline{41}$ and sodium ${ }^{42}$ is absent from dense hydrogen). In addition, the $s$ orbital electrons, the only one that is relevant in hydrogen, are always well described by LDA even at ambient conditions.

On the other hand, although a reliable convergence of the DFT total energy to within $3 \mathrm{meV}$ per proton has been achieved, a concern that whether DFT can be applied to dense hydrogen beyond insulator-metal transition might still exist because it is well known that DFT underestimates band gap and then the transition pressure. It is a severe problem for electronic structure properties, but has limit impacts on the total energy and geometry features. Theoretically DFT only ensures the correct charge density and the total energy, rather than the quasi-particle levels that are introduced via Kohn-Sham ansatz. For example in an extreme case where the exact exchange-correlation functional were available, DFT would become rigorous and predict an exact charge density and total energy, whereas the band structure still cannot be generally guaranteed theoretically. ${ }^{61}, 63$ On the other hand, there are many examples in the applications of DFT to condensed matters where the band structure given by DFT is wrong but the energetics and atomic structure are still within an 


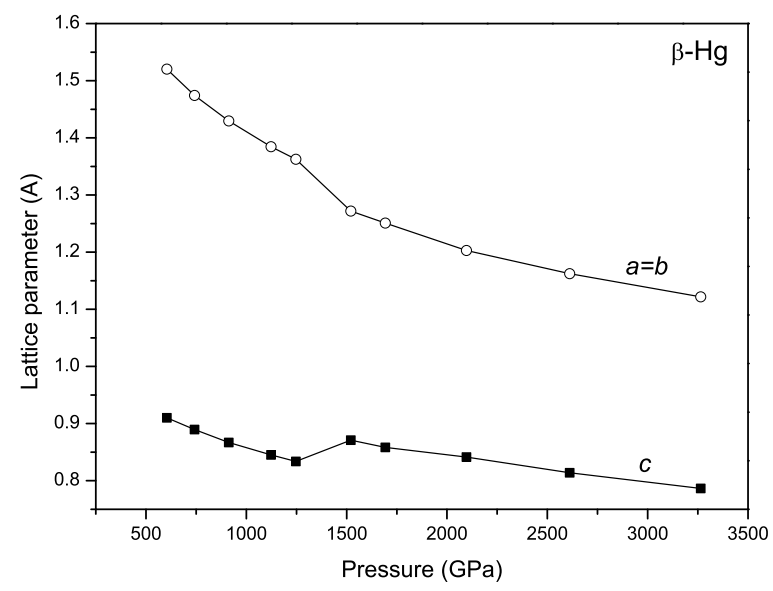

FIG. 14. Variation of the tetragonal lattice parameters of $\beta$-Hg under compression, where the shortest lattice vector $c$, which indicates the bond length of the molecular chain, is always longer than the ambient $\mathrm{H}_{2}$ value of $0.74 \AA$.

acceptable precision. Therefore the underestimation of the band gap of dense hydrogen by DFT does not present as a serious issue to what we concerned here. The intrinsic error in the total energy and structural features comes from the GGA approximation of the energy functional, and should be similar for insulator and metallic phases as long as the spatial variation of the charge density is similar.

It also needs to point out that near the transition point from insulator to metallic state, where the band gap begins closing up and electrons jump abruptly from localized to delocalized orbitals, DFT might underestimate the transition pressure,,$\frac{5}{2}$ presumably due to self-interaction errors in exchange-correlation functional. But far away from this transi-

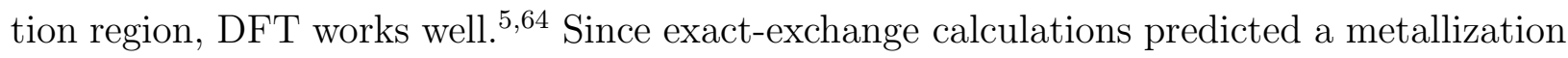
pressure of hydrogen at $400 \mathrm{GPa}, \stackrel{65}{\frac{6}{3}}$ much lower than the pressure range interested here, we estimate that the influence on the total energy and structure features due to the errors in delocalization of electrons occurred at low pressures should be small.

\section{Appendix B: Justification to the pseudopotential}

We used a PAW pseudopotential that is harder than the standard one, which is specially designed to account for the possible short interatomic distance under ultra-high compressions. The performance of this potential was checked by comparing the bond length and 
binding energy of $\mathrm{H}_{2}$ dimer with those of other potentials and all-electron calculations. The low-lying structures and the corresponding transition pressures below $400 \mathrm{GPa}$ as reported in Ref.17 were also perfectly reproduced. This verified that the hard potential works correctly. Its applicability to higher pressure range can be generally guaranteed as long as the shortest interatomic distance is greater than twofold of the outmost cutoff radius $r_{m}$ of the potential, which defines the atomic spheres (augmentation regions) where the pseudo wave function takes effects. This precondition ensures the correct wave-function shape near the atomic spheres. For comparison, $r_{m}=0.42 \AA$ for the hard potential and $r_{m}=0.58 \AA$ for the standard potential of hydrogen. In our studied pressure range here, no low-lying structure has a shortest interatomic distance less than the ambient hydrogen molecule bond length of $0.74 \AA$. For example, Fig 14 shows the variation of the lattice parameters of $\beta$ - $\mathrm{Hg}$ with pressure, where $c$ indicates the shortest bond length and is always greater than $0.74 \AA$. The overlapping between atomic spheres of dense hydrogen is less than that of $\mathrm{H}_{2}$ molecule at ambient conditions. Therefore the application of the PAW potential of hydrogen to high pressures does not present as a difficult issue. It is worthwhile to point out that for am-

bient $\mathrm{H}_{2}$, there is a little overlap of the pseudopotential regions on nearest neighboring hydrogen atoms. In principle these spheres shouldnot overlap, but a bit of overlap/softnessof-the-potential tradeoff is usually acceptable. Especially when spherical Bessel functions were used to construct the PAW potential, as implemented in VASP code, a large overlap between the atomic spheres is allowed..$^{32}$

An overall examination of the validity of the PAW potential is illustrated in Fig, 15, where the VASP result is compared directly with that of the all-electron method calculated with WIEN2k code. ${ }^{66}$ Both calculations used the same PBE exchange-correlation energy functional. We can see from this figure that within a wide range of pressure up to $4 \mathrm{TPa}\left(r_{s} \approx\right.$ 0.9), both methods give almost the same energy difference between high-symmetric BCC and FCC structures (the maximum deviation is less than $0.4 \mathrm{meV}$ per proton), reflecting the fact that the error introduced by PAW potential is insignificant.

\section{Appendix C: Structure of some meta-stable phases}

The structural information (including both lattice parameters and atomic coordinates) of some meta-stable multi-atomic molecular phases and the degenerate groundstate $F d d d$ 


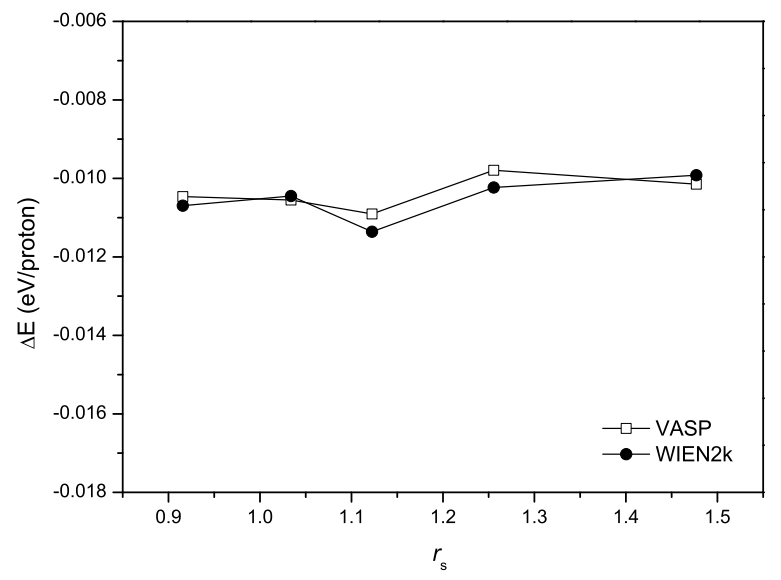

FIG. 15. Comparison of the energy difference between FCC and BCC phases as a function of density calculated using PAW pseudopotential method with VASP from that of all-electron results of WIEN2k.

at selected pressures are listed in table \. 
TABLE I. Structure of some low-lying phases. Only the fractional coordinates of symmetry inequivalent atoms are reported. The numbers of atoms in a primitive cell are given. Immm is a coarse but robust (with greater tolerance) orthorhombic representation of $C 2 / m$ structure. For $\beta-\mathrm{Hg}$, a tetragonal distortion of the BCC cubic cell is adopted.

\begin{tabular}{|c|c|c|c|c|c|c|c|}
\hline \multirow{3}{*}{$\begin{array}{l}\text { space group } \\
\sharp \text { atoms } \\
C 2 / m(1)\end{array}$} & \multirow{3}{*}{$\begin{array}{c}\text { pressure } \\
(\mathrm{GPa}) \\
650\end{array}$} & \multicolumn{3}{|c|}{ lattice parameter } & \multicolumn{3}{|c|}{ atomic coordinates } \\
\hline & & $\left(\AA,{ }^{\circ}\right)$ & & & \multicolumn{3}{|c|}{ (fractional) } \\
\hline & & $a=3.178 \quad b=1.203$ & $c=2.395$ & $\mathrm{H} 1$ & 0.9999 & 0.0000 & 0.8300 \\
\hline 3 & & $\alpha=\gamma=90.00$ & $\beta=137.63$ & $\mathrm{H} 2$ & 0.5000 & 0.0000 & 0.5000 \\
\hline Immm & & $a=2.142 \quad b=1.203$ & $c=2.395$ & $\mathrm{H} 1$ & 0.5000 & 0.0000 & 0.6766 \\
\hline 3 & & $\alpha=\beta=\gamma=90$ & & $\mathrm{H} 2$ & 0.0000 & 0.0000 & 0.5000 \\
\hline$C 2 / m(2)$ & 1000 & $a=3.180 \quad b=1.101$ & $c=2.711$ & $\mathrm{H} 1$ & 0.0006 & 0.0000 & 0.3376 \\
\hline 3 & & $\alpha=\gamma=90.00$ & $\beta=147.51$ & $\mathrm{H} 2$ & 0.5000 & 0.0000 & 0.0000 \\
\hline Immm & & $a=1.708 \quad b=1.101$ & $c=2.711$ & $\mathrm{H} 1$ & 0.0000 & 0.0000 & 0.0000 \\
\hline 3 & & $\alpha=\beta=\gamma=90$ & & $\mathrm{H} 2$ & 0.5000 & 0.0000 & 0.1674 \\
\hline Pmmn & 1104 & $a=2.349 \quad b=1.110$ & $c=1.877$ & H1 & 0.8170 & 0.5000 & 0.6886 \\
\hline 6 & & $\alpha=\beta=\gamma=90$ & & $\mathrm{H} 2$ & 0.0000 & 0.5000 & 0.1087 \\
\hline$\beta-\mathrm{Hg}(I 4 / \mathrm{mmm})$ & 1012 & $a=b=1.408$ & $c=0.856$ & H1 & 0.0000 & 0.0000 & 0.0000 \\
\hline 1 & & $\alpha=\beta=\gamma=90$ & & & & & \\
\hline$F d d d$ & 533 & $a=3.042 \quad b=1.832$ & $c=1.596$ & $\mathrm{H} 1$ & 0.7500 & 0.7500 & 0.2500 \\
\hline 2 & & $\alpha=\beta=\gamma=90$ & & & & & \\
\hline
\end{tabular}

